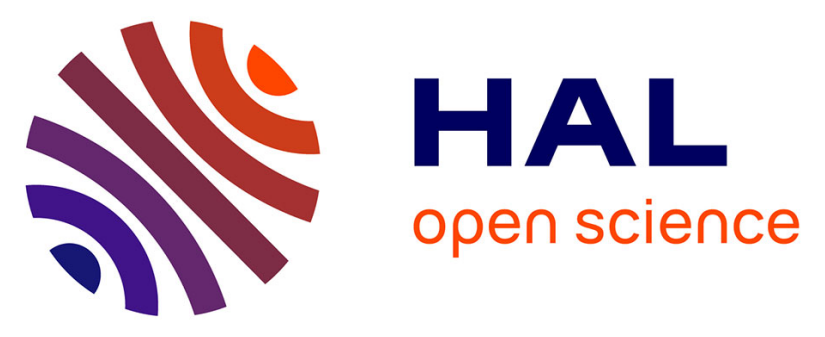

\title{
Characterization and transcriptional profiles of three Spodoptera frugiperda genes encoding cysteine-rich peptides. A new class of defensin-like genes from lepidopteran insects?
}

Anne Nathalie Volkoff, Janick Rocher, Emmanuelle d'Alençon, M. Bouton, Igor Landais, Enrique Quesada-Moraga, Alain Vey, Philippe Fournier, Kazuei Mita, Gerard Devauchelle

\section{- To cite this version:}

Anne Nathalie Volkoff, Janick Rocher, Emmanuelle d'Alençon, M. Bouton, Igor Landais, et al.. Characterization and transcriptional profiles of three Spodoptera frugiperda genes encoding cysteine-rich peptides. A new class of defensin-like genes from lepidopteran insects?. Gene, 2003, 319, pp.43-53. 10.1016/S0378-1119(03)00789-3 . hal-02678487

\section{HAL Id: hal-02678487 https://hal.inrae.fr/hal-02678487}

Submitted on 31 May 2020

HAL is a multi-disciplinary open access archive for the deposit and dissemination of scientific research documents, whether they are published or not. The documents may come from teaching and research institutions in France or abroad, or from public or private research centers.
L'archive ouverte pluridisciplinaire HAL, est destinée au dépôt et à la diffusion de documents scientifiques de niveau recherche, publiés ou non, émanant des établissements d'enseignement et de recherche français ou étrangers, des laboratoires publics ou privés. 


\title{
Characterization and transcriptional profiles of three Spodoptera frugiperda genes encoding cysteine-rich peptides. A new class of defensin-like genes from lepidopteran insects?
}

\author{
Anne-Nathalie Volkoff ${ }^{\text {a** }}$, Janick Rocher ${ }^{\mathrm{a}}$, Emmanuelle d'Alençon ${ }^{\mathrm{a}}$, Martine Bouton, \\ Igor Landais $^{\mathrm{a}}$, Enrique Quesada-Moraga ${ }^{\mathrm{b}}$, Alain Vey ${ }^{\mathrm{a}}$, Philippe Fournier ${ }^{\mathrm{a}}$, \\ Kazuei Mita ${ }^{c}$, Gérard Devauchelle ${ }^{a}$ \\ ${ }^{a}$ Laboratoire de Pathologie Comparée, UMR 5087 INRA-CNRS/Universite Montpellier II, 30380 Saint-Christol-les-Alès, France \\ ${ }^{\mathrm{b}}$ Unidad de Entomología Agrícola, Departamento de Ciencias y Recursos Agrícolas y Forestales, ETSIAM, Universidad de Córdoba, Apdo 3048, \\ Córdova 14080, Spain \\ c Laboratory of Insect Genome, National Institute of Agrobiological Sciences, Tsukuba, Ibaraki 305-8634, Japan
}

Received 12 March 2003; received in revised form 16 June 2003; accepted 24 June 2003

Received by G. Pesole

\begin{abstract}
The present work describes sequence and transcription of three Spodoptera frugiperda genes encoding 6-cysteine-rich peptides. Sequence alignments indicate that the predicted peptides belong to the insect defensin family, although phylogenetic analyses suggest they form a cluster distinct from that of other neopteran insect defensins. The three genes were identified in a non-immune-challenged Sf9 cells cDNA (DNA complementary to RNA) library (Landais et al., Bioinformatics, in press) and were named spodoptericin, Sf-gallerimycin and Sf-cobatoxin. Spodoptericin is a novel defensin-like gene that appears to be weakly up-regulated following injection of bacteria and fungi. Interestingly, no sequence motif clearly homologous to cis regulatory element involved in the regulation of antimicrobial genes was found. An homologue of the spodoptericin gene was identified in the SilkBase Bombyx mori cDNA library. Sf-gallerimycin is related to the Galleria mellonella gallerimycin gene and is induced after immune challenge by injection of bacteria in the larval fat body as well as in hemocytes. In silico analysis of the sequence upstream from the cDNA reveals the presence of at least one motif homologous to a nuclear factor kappaB (NF- $\kappa \mathrm{B})$ binding site. Finally, Sf-cobatoxin is related to the G. mellonella cobatoxin-like gene. Despite high levels of constitutive expression compared to the two previous genes, transcription of Sf-cobatoxin is increased after immune, in particular, bacterial challenge. We therefore confirm that these three genes encode potential candidate molecules involved in $S$. frugiperda innate humoral response.
\end{abstract}

(C) 2003 Elsevier B.V. All rights reserved.

Keywords: Insect immunity; Antimicrobial peptides; Sf9; cDNA library

Abbreviations: aa, amino acid(s); BAC, bacterial artificial chromosome; cDNA, DNA complementary to RNA; CSab, cysteine-stabilized alpha beta motif; EST, expressed sequence tag; nt, nucleotide(s); Imd, immune deficiency (pathway); kb, kilobase(s); $\mathrm{kDa}$, kilodalton(s); ORF, open reading frame; PFAM, protein families database of alignments; PCR, polymerase chain reaction; Prosite, database of protein families and domains; SCOP, database of structural classification of proteins; Brn-2, POU factor Brain-2-; CdxA, caudal homeobox gene A; Dfd, Deformed; GATA-1, GATA-binding factor 1; IL-6 RE-BP, interleukin-6 response element binding protein; c-Myb, cellular homolog of avian myeloblastosis virus oncogene; NF-кB, nuclear factor kappaB; Oct-1, octamer-binding factor 1; UTR, untranslated region.

* Corresponding author. Tel.: +33-4-66-78-37-26; fax: +33-4-66-5246-99.

E-mail address: volkoff@ensam.inra.fr (A.-N. Volkoff).

\section{Introduction}

In insects, cysteine-rich peptides constitute a diverse and widely distributed family of defense molecules against bacterial and fungal infections (Dimarcq et al., 1998). The insect defensins, which belong to this family, are peptides with a cysteine-stabilized $\alpha \beta(\mathrm{CS} \alpha \beta)$ motif involving six conserved cysteine residues that confers to the molecules high stability and resistance to the proteases. Insect defensins present sequence similarities with mammalian defensins but they are structurally unrelated since the latter consist of $\beta$-sheets and lack the $\alpha$-helix characteristic of insect defensins (Dimarcq et al., 1998). In all multicellular 
organisms, including humans and plants, defensins appear to have a wide range of actions directed against various microbes (Hoffmann et al., 1999). Mammalian defensins disrupt microbial membranes and chemically attract $\mathrm{T}$ cells whereas plant defensins are primarily directed against fungal pathogens. In Drosophila, defensins have been shown to form voltage-dependent ion channels in Grampositive cells (Cociancich et al., 1993).

Insect defensins are generally cationic peptides composed of 34-46 residues, with molecular masses ranging from 2 to 6 kilodaltons $(\mathrm{kDa})$. Initially discovered in diptera (Matsuyama and Natori, 1988), defensins have since been described in many neoptera insect orders. Most act against a broad spectrum of Gram-positive bacteria but have little effect on Gram-negative bacteria and fungi (Dimarcq et al., 1998). Defensin-like peptides have recently been identified in lepidoptera. The best known of these is heliomicin, a defensin-like peptide identified in the moth Heliothis virescens. Interestingly, heliomicin has mainly antifungal properties and is more related to the Drosophila antifungal drosomycin than to defensins (Lamberty et al., 1999).

Studies on the immune system of Drosophila suggest that innate immunity is an ancient defense mechanism (Hoffmann et al., 1999). In insects, all promoters of immune-inducible peptide genes contain responsive elements homologous to those involved in the regulation of genes-encoding acute-phase proteins in mammals. The most prominent and often mandatory are binding sites for transcriptional activators of the Rel family. In Drosophila, the functional importance of the $\mathrm{kB}$-related sequences has been demonstrated for several antibacterial and antifungal genes (Meister et al., 1994). Genetic and functional studies have shown that production of subsets of peptides against different pathogens is based on the activation of two distinct but maybe cross-talking signaling cascades - the Toll dorso-ventral-signaling pathway and the immune deficiency (Imd) pathway (reviewed in Khush et al., 2001; Hoffmann and Reichhart, 2002). For example, the Drosophila defensin gene requires both Toll and Imd pathways for full inducibility following immune challenge (Lemaitre et al., 1997). In lepidopterans, kB-like sequences occur in the promoter regions of the B. mori antibacterial cecropins, attacin and lebocins genes (Ponnuvel and Yamakawa, 2002), suggesting that antimicrobial lepidopteran genes are regulated through similar pathways. To date, however, there is no published information on lepidopteran genes encoding cysteine-rich peptides such as defensins.

In this study, we used a Sf9 cDNA (DNA complementary to RNA) library constructed from non-immune-challenged cells recently made available to us (Landais et al., 2003) to detect defensin-like peptide genes in lepidopterans and to investigate their transcriptional regulation. Genes encoding three cysteine-rich defensin-like peptides expressed in the lepidopteran Spodoptera frugiperda were identified from the Sf9 cells of this cDNA library. The first encodes a novel peptide, named spodoptericin, distinct from previously described lepidopteran defensin-like peptides such as heliomicin and the Mamestra brassicae defensin (GenBank AAL69980). The other two are related to recently identified cysteine-rich peptides of the greater wax moth Galleria mellonella and resemble scorpion potassium-channel blocking peptides named cobatoxins (Seitz et al., 2003). To assess the role of these three predicted peptides in the immune response in S. frugiperda, we conducted a transcription analysis of the genes following immune-challenge with germs such as bacteria and/or the entomopathogenic fungus Beauveria bassiana. We also analyzed the regions upstream from the transcription start for the genes encoding two of these peptides in order to identify sequence motifs homologous to cis regulatory elements involved in the regulation of genes encoding antimicrobial peptides.

\section{Material and methods}

\subsection{Sf9 cells cDNA expression library}

The clones used are indicated in Fig. 1A and belong to Sf9 cells (ATCC CRL1711) cDNA expression library (Landais et al., 2003). The clones \#Sf9L00498 and Sf9L07974 correspond to a $S$. frugiperda defensin-like gene we named spodoptericin as no previously described homologues were found. Based on similarity with previously described genes, the clones \#Sf9L06137 and Sf9L06454 correspond to a gene we named Sf-gallerimycin. Four clones, \#Sf9L00509, Sf9L02701, Sf9L03867 and Sf9L06099 correspond to genes we named Sf-cobatoxins. Sf9L00509 and Sf9L02701, as well as Sf9L03867 and Sf9L06099, are overlapping clones and all four clones have a common 3 '-end sequence. For clarification purposes, the nomenclature of the clones will be simplified in all subsequent text. For example, SF2701 will be used instead of Sf9L02701.

\subsection{Isolation of the complete coding sequence}

To isolate the genomic sequences, polymerase chain reactions (PCRs) were conducted using Sf9 genomic DNA as a template and internal primers within the cDNA sequences. The primer pairs were SF7974_For 5'-GGT GTC GGC TTG TCT GAT ACA-3' (17 nt after the ATG codon), and SF7974_Rev 5'-GCA GCT ACA TGT GTG ACT GAC-3' (4 nt before the TAA codon) for the spodoptericin gene; SF6454_For 5'-AAG GTT TCA GTC ATG AAG GCT TGC-3' (12 nt before the ATG codon), and SF6454_Rev 5'TAC AAA CAT GGC AAG ATG GAG AGC-3' (37 nt after the TAG codon) for the Sf-gallerimycin gene; and SF6099_For 5'-AGC GTT GTT GAC TTT TTC ACC TCA-3' (70 nt before the ATG codon), and SF6099_Rev 5'GGC TGA AAC GCA GTT TCC ATA TTT-3' (22 nt before 


\begin{tabular}{|c|c|c|c|}
\hline EST clones & BlastX best match & E-score & gene name \\
\hline Sf9L00498 (AY238438,322bp); Sf9L07974 (AY238439,561bp) & Palomena prasina defensin, GenBank\#P80407 & 2,4 & spodoptericin \\
\hline Sf9L06137 (AY238440,375bp); Sf9L06454 (AY238440,375bp) & Galeria mellonella gallerimycin, GenBank\#AF453824 & e-12 & Sf-gallerimycin \\
\hline Sf9L00509 (AY238441,450bp); Sf9L02701 (AY238442,504bp) & G. mellonella cobatoxin-like, GenBank\#AF394590 & 9.e-7 & Sf-cobatoxin a \\
\hline Sf9L03867 (AY238443,319bp); Sf9L06099 (AY238444,568bp) & idem & & Sf-cobatoxin $b$ \\
\hline
\end{tabular}

A

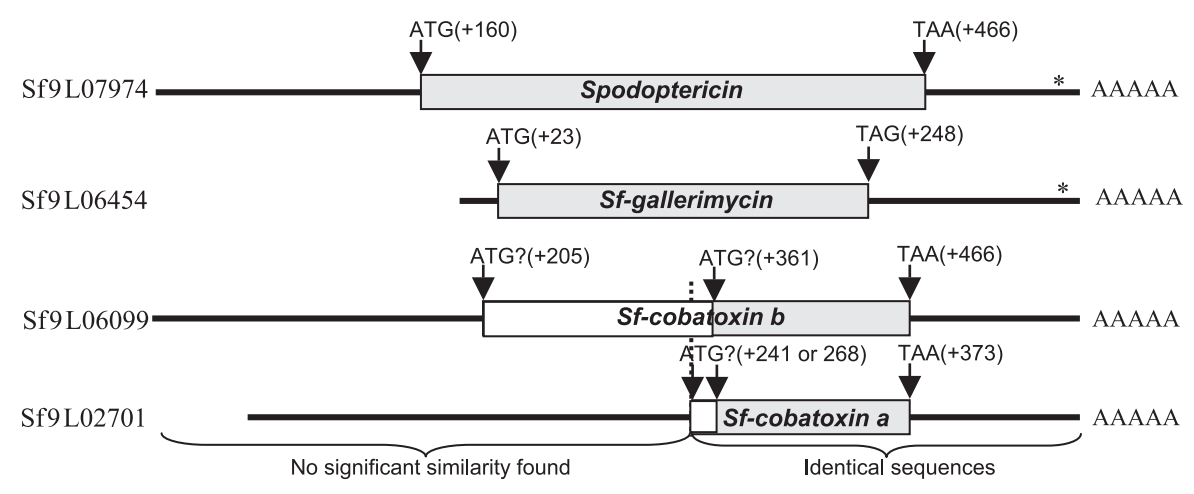

B

\begin{abstract}
Spodoptericin (SF7974)

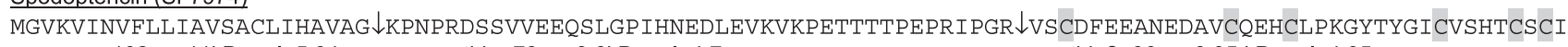
precursor:102aa; $11 \mathrm{kDa} ; \mathrm{pl}=5.04 \quad$ propeptide : $78 \mathrm{aa} ; 8.6 \mathrm{kDa} ; \mathrm{pl}=4.7$ peptide? : $36 \mathrm{aa} ; 3.95 \mathrm{kDa} ; \mathrm{pl}=4.35$

Sf-gallerimycin (SF6454)

MKACVVLAVLLVAFAVATSTA $\downarrow$ DLGHTEASLRVRRETIRGPEFP $\downarrow_{\text {NRCVFYECIASCRQRGYKSGGYCTINGCQCLR }}$

Pre-: $75 \mathrm{aa} ; 8.2 \mathrm{kDa}$; $\mathrm{pl}=9 \quad$ pro- : $54 \mathrm{aa} ; 6.2 \mathrm{kDa}$; $\mathrm{pl}=8.9$ peptide? : $32 \mathrm{aa} ; 3.6 \mathrm{kDa}$; $\mathrm{pl}=8.9$

Sf-cobatoxin (SF6099) predicted peptide (by analogy with G. mellonella): $35 \mathrm{aa} ; 3.9 \mathrm{kDa} ; \mathrm{pl}=8.64$

MKLILFVVCAMVMAAYA $\downarrow$ VEVISVDDLLRAYDVSHNKIDGESVKMATEALYVPMSL $\downarrow$ RACTSSACNFVCKFLGYKYGNCVSAETCRCYS

Pre-:87aa; $9.6 \mathrm{kDa} ; \mathrm{pl}=6.5$ propeptide : $70 \mathrm{aa} ; 7.7 \mathrm{kDa} ; \mathrm{pl}=5.6 \quad$ !! peptide? : $32 \mathrm{aa} ; 3.5 \mathrm{kDa} ; \mathrm{pl}=8.65$

!SF2701 peptide: $44 \mathrm{aa} ; 4.8 \mathrm{kDa} ; \mathrm{pl}=8.3$
\end{abstract}

Fig. 1. Sf9 cells cDNAs encoding cysteine-rich peptides. (A) The table indicates the EST clone references, their best BlastX match and score and the deduced name of the corresponding genes. A schematic representation of the longest clone is shown below the table. Boxes represent the open reading frames. For Sf-cobatoxins, shaded box correspond to the ORF predicted if sequence is compared to the G. mellonella cobatoxin. The start and stop codons are indicated by vertical arrows, position +1 corresponds to the start of the EST clone. Asterisk indicates a polyA signal. (B) Predicted amino acid sequences. The position of possible cleavage sites is indicated by a vertical arrow. For each peptide [precursor (pre-), pro-peptide (pro-) and putative mature peptide (peptide)], the length, the calculated molecular weight and the theoretical pI are indicated. For Sf-cobatoxin, sequence of the peptide predicted to be encoded by SF2701 (SF2701 peptide) is underlined, and the most probable sequence for this peptide (predicted peptide, by analogy with G. mellonella) is in bold characters. The conserved six cysteine residues are highlighted.

the TAA codon) for the Sf-cobatoxin gene. PCR was conducted with Taq DNA polymerase according to standard procedure. The obtained amplimers were cloned in pGEMTEasy vector (Clontech) and sequenced (GenomeExpress, France).

\subsection{Analysis of the gene copy number by Southern blot} analysis

The gene copy number was analyzed by Southern blot analysis of (1) S. frugiperda genomic DNA digested with restriction enzymes absent from the known sequence (Pst $\mathrm{I}$ or HindIII) and (2) a S. frugiperda bacterial artificial chromosome (BAC) library (potential $10 \times$ coverage, d'Alençon et al., in preparation). Genomic DNA was extracted from $S$. frugiperda 4 th instar larvae according to standard procedures. Hybridizations were conducted as described previously (Volkoff et al., 1999; d'Alençon et al., in preparation), using the cDNA inserts as probes.

\subsection{Isolation of the spodoptericin and Sf-gallerimycin genes upstream sequence}

Cloning of the region upstream from the spodoptericin cDNA was accomplished by inverse PCR. Digestion of the genomic Sf9 DNA with the restriction enzyme SmaI resulted in a 2-kbp hybridization band with the spodoptericin probe in preliminary Southern blot analysis. SmaI digested genomic DNA was then recircularized and the ligation product used as template for a PCR using the primers SF7974_invF 5'-ATA CCT GGC GTT GTG GTG GTT TCT G-3' and SF7974_invR 5'-GAA GTA GCG GCT GCG ATA AAC CTC TTA $-3^{\prime}$. The resulting PCR product was cloned in pGEM-TEasy vector and approximately $790 \mathrm{bp}$ were sequenced upstream from the cDNA sequence.

To obtain Sf-gallerimycin upstream sequence, two positive BAC clones (BAC61_G18 and BAC68_H5) were sequenced using the gene specific primer BAC6454 5'-GAA CCA CGC AAG CCT TCA TGA CTG-3' (5 nt upstream the 
ATG codon, underlined). Approximately $600 \mathrm{bp}$ were sequenced upstream from the Sf-gallerimycin cDNA sequence.

\subsection{Transcription analysis by Northern blot}

To collect total RNA samples, dissected fat body cells were placed directly into $0.5 \mathrm{ml}$ of Trizol reagent (Gibco). Hemocyte samples were collected by bleeding larvae into $0.75 \mathrm{ml}$ of Trizol reagent. To analyze transcription during $S$. frugiperda development, total RNA was extracted from whole larvae of 1st, 2nd and 3rd instars, and from fat body of 4th, 5th, pupa and adult instars.

Hybridizations were conducted as described previously (Volkoff et al., 1999), using the cDNA inserts as probes. The same membrane was used for all three probes by stripping the membrane after each hybridization. A S. frugiperda actin probe was generated by PCR from Sf9 DNA, using the forward primer 5'-CAA CTG GGA CGA CAT GGA GAA GAT- $3^{\prime}$ and the reverse primer $5^{\prime}$-CCA CCG ATC CAT ACG GAG TAT TTC-3' and was used as an internal standard.

\subsection{Microrganisms used for immunization assays}

The bacterial strains used were the Gram-positive Bacillus subtilis (strain 168, gift from E. Le Chatelier, INRA Jouy-enJosas), and the Gram-negative Escherichia coli (strain JM83). The fungus strain used was the entomopathogenic B. bassiana (strain 80.2 from our laboratory's collection). The polydnavirus used was HdIV, which is associated with the ichneumonid wasp Hyposoter didymator (Volkoff et al., 1999).

\subsection{Immunization of insects}

In a first assay, 1-day old $S$. frugiperda 5 th instar larvae were immune-challenged by injection of either $8 \times 10^{2} B$. bassiana conidia or $8 \times 10^{5}$ live bacteria (mix of $B$. subtilis and $E$. coli) per insect. For bacterial challenge, we chose to use a mix of both Gram-negative and Gram-positive bacteria, as each bacterial type activates different regulation pathways: the first activates the Imd pathway whereas the second activates preferentially the Toll pathway. Larvae were infected with HdIV as described in Volkoff et al. (2002) and immune-challenged $4 \mathrm{~h}$ after infection. HdIV infection of $S$. frugiperda larvae was controlled using a probe composed of viral cDNAs. Hemocytes and fat bodies were recovered 24 and $48 \mathrm{~h}$ after immune challenge $(n=5$ larvae per assay). For fat bodies, two repeats were done for the Sf-gallerimycin and Sf-cobatoxin probes. For the spodoptericin probe, two additional assays were done with individual larvae.

In a second assay, 1-day old $S$. frugiperda 5 th instar larvae were injected with two suspensions of $B$. bassiana conidia in PBS, corresponding to either $8 \times 10^{2}$ or $8 \times 10^{3}$ conidia per insect. Fat bodies were recovered 24 and $48 \mathrm{~h}$ after injection $(n=5$ larvae per assay).

\subsection{Immunization of cells in culture}

The Sf9 cells were treated with a cell medium suspension of previously heat-inactivated E. coli, B. subtilis and $B$. bassiana conidia or with a suspension of LPS and zymozan. Samples were collected 24 and $48 \mathrm{~h}$ after treatment.

\subsection{Sequence analysis}

Comparisons of sequences in nonredundant databases were carried out using BLASTX Sequence Similarity Searches (http://www.ncbi.nlm.nih.gov). Molecular weight of the deduced proteins was calculated with the ProtParam tool (http://www.expasy.ch/cgi-bin). Comparison with protein family databases was performed with the Family Pairwise Search (http://fps.sdsc.edu). Transcription factor binding consensus sites in the putative promoter regions were identified using TFSEARCH (http://molsun 1.cbrc. aist.go.jp/ research/db/TFSEARCH.html) software, with the threshold score varying from 70 to 85 (default). Sequence were aligned and bootstrapped neighbor-joining trees were calculated using ClustalX and TreeView software, respectively.

\section{Results and discussion}

3.1. Isolation of S. frugiperda cDNAs encoding predicted cysteine-rich peptides and in silico analysis of the predicted amino acid sequences

Analysis of the non-immune challenged Sf9 cells cDNA library revealed the existence of several clones related to three distinct genes encoding cysteine-rich peptides (Fig. 1A). One is a novel gene first described in the present work that we named spodoptericin. Based on their similarity with other genes, the other two were named Sf-gallerimycin and Sf-cobatoxin. Only the longest and most probable fulllength cDNA will be discussed here: SF7974 (spodopterricin), SF6454 (Sf-gallerimycin) and SF6099 (Sf-cobatoxin). The clones SF6099 and SF2701 share the terminal $280 \mathrm{nt}$ in the $3^{\prime}$ end of the sequence (Fig. 1A, diagram) but differ in their 5 'regions. Indeed, comparison of the divergent 5 'regions show no significant similarity between the two sequences. As opposed to the two other cDNAs, no polyadenylation signal was found in the $3^{\prime}$ untranslated region (UTR) region of the Sf-cobatoxin cDNAs. However, since only the open reading frame (ORF) sequence is available for the G. mellonella cobatoxin-like (GenBank AF453824), we do not know if the absence of a polyadenylation signal is a feature of this gene family. In all $S$. frugiperda expressed sequence tag (EST) clones, a putative ORF is present (diagram, Fig. 1A; sequences in Fig. 1B). However, the ORFs for the two Sf-cobatoxin cDNAs may be shorter than that predicted. Indeed, if the sequences are compared with their G. mellonella homologue, the actual ORF may correspond only to the last $105 \mathrm{nt}$ of the 
sequences and be the same for the two clones (shaded boxes in diagram, Fig. 1A).

The three $S$. frugiperda predicted peptides each contains six cysteine residues that are probably involved in intramolecular disulfide bonds. They all present similarities with the arthropod defensins signature (Prosite signature: $\mathrm{C}-\mathrm{x}(2,3)-$ [HN]-C-x(3,4)-[GR]-x(2)-G-G-x-C-x(4,7)-C-x-C) when compared with domain databases [database of protein families and domains (Prosite), protein families database of alignments (PFAM) and database of structural classification of proteins (SCOP)]. In contrast to other known insect defensins, they also present to some extent the scorpion short toxins signature (Prosite signature: $\mathrm{C}-\mathrm{x}(3)-\mathrm{C}-\mathrm{x}(6,9)-[\mathrm{GAS}]-$ K-C-[IMQT]-x(3)-C-X-C). Although unusual, the similarity between scorpion toxins and insect defensins is not surprising: both use a similar CS $\alpha \beta$ motif folding pattern, suggesting that these molecules may have a common ancestor with a similar structural organization. Among the S. frugiperda predicted peptides, only spodoptericin presents a stretch of 10 amino acids (aa) between the first two cysteine residues, comparable to what is found in other insect defensins (alignment in Fig. 2A). Nevertheless, based on their amino acid sequences, the three $S$. frugiperda predicted peptides might be considered as defensin-like peptides.

Sequence and features of the predicted peptides are shown in Fig. 1B. As mentioned previously, Sf-cobatoxin is probably shorter than predicted if compared to the sequence available for the $G$. mellonella homologue (57\% identity). Data in Fig. 1B are given for all predicted sequences, although only the most probable 35 aa predicted Sf-cobatoxin peptide will be discussed here (Fig. 1B, bolded sequence). Spodoptericin and Sf-gallerimycin contain a putative hydrophobic signal sequence and are most likely cleaved. For all three proteins, other potential cleavage sites located two amino acids before the first cysteine residue were determined by aligning the sequences with known insect defensins (Fig. $1 \mathrm{~B}$, peptides). Cleavage would result in the formation of predicted mature peptides of 36, 32 and 32 amino acids for spodoptericin, Sf-gallerimycin and Sf-cobatoxin, respectively, which is consistent with the length of other known insect

A

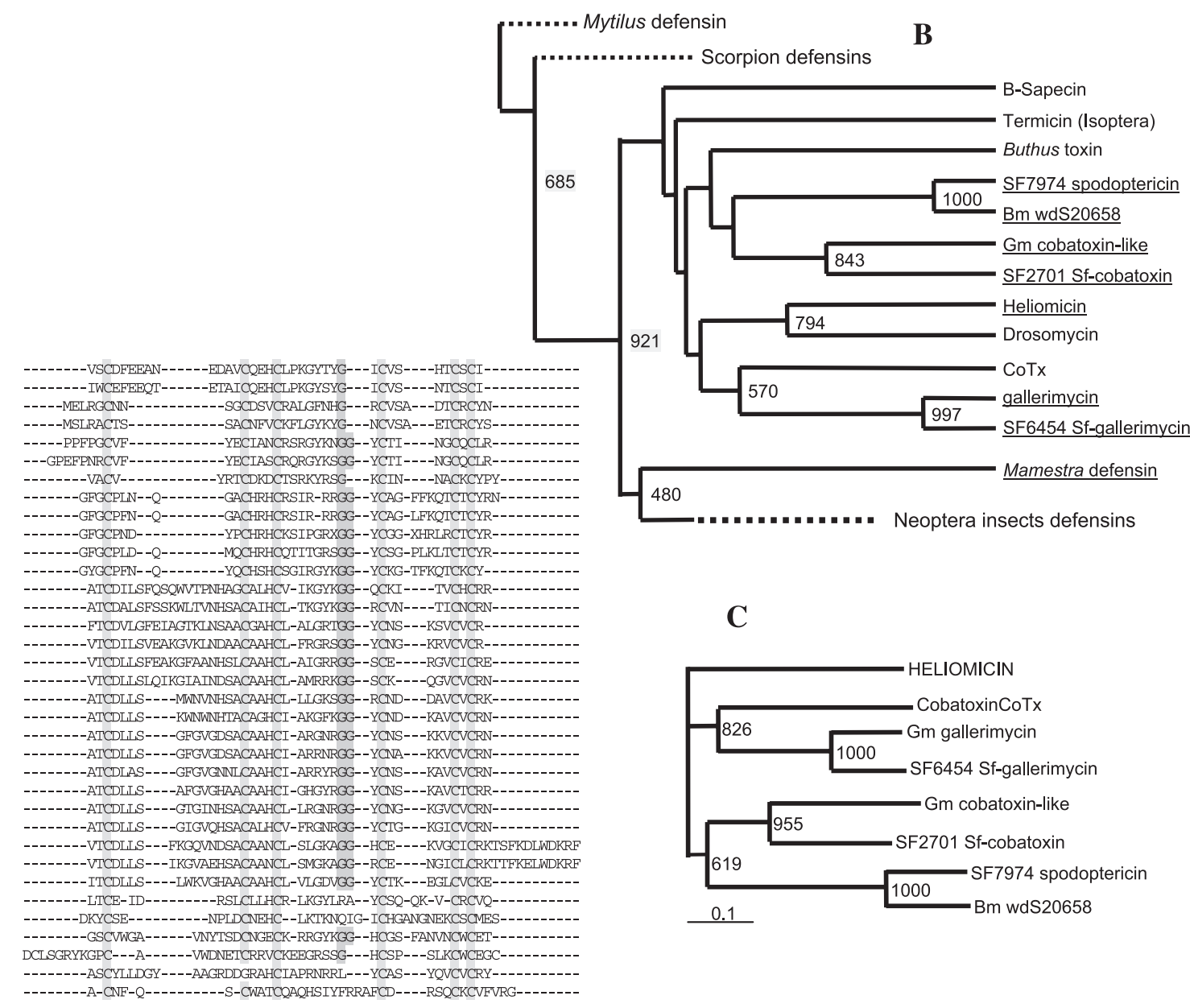

480
Spodopdericin SF7974 Bombyx mori wds20658

G.m cobatoxin-like

Sf-cobatoxin SF270

Andronoctus australis

Mytilus sp.

Aeschna cyanea

Omithodoros sp.

Pyrrhocoris apteru

Zaphobas atratus

Tenebrio molitor

Ilomyrina dichotoma

Stomoxys calcitrans

Aedes aegypti

Anopheles gambi

Phlebotomis sp.

Phormia terranova

Sarcophaga peregrina

Apis mellifera

Stomoxys calc

Sarcophaga peregrina $\mathrm{B}$

Drosomycin

veudacanthotermes

Fig. 2. Phylogenetic analysis of cysteine-rich defensin-like peptides. (A) ClustalX alignment of several arthropod defensins, two scorpion toxins (underlined) and the lepidoptera peptides using the cysteines as landmarks (highlighted). (B) Simplified phylogenetic tree generated from the sequences aligned in (A) using the neighbor-joining method with the mussel Mytilus sp. defensin defined as outgroup. (C) Neighbor-joining tree generated from the lepidopteran cysteine-rich peptides and cobatoxin (CoTx) sequences with heliomicin defined as an outgroup. Boostrap values (of 1000 replicates) are indicated at the nodes of branches when $>480$. The scale at the bottom of $(\mathrm{C})$ corresponds to $10 \%$ divergence between sequences. 
defensins. Based on calculated pIs, Sf-gallerimycin and Sfcobatoxin are predicted to be cationic, with or without cleavage, which is in agreement with insect defensins. Interestingly, spodoptericin is predicted to be anionic. Cationic peptides are more often antibacterial whereas neutral peptides are more often antifungal (Hetru, personal communication), therefore, spodoptericin is a novel anionic cysteinerich peptide for which the function is still unknown.

\subsection{Phylogenetic analysis of the lepidopteran defensin-like peptides}

To analyze the lepidopteran defensin-like family, a large array of arthropod defensins and some scorpion toxins were aligned using ClustalX then manually using the cysteines as landmarks (Fig. 2A). Analysis of the Bombyx mori SilkBase EST library (http://www.ab.a.u-tokyo.ac.jp/silkbase/; Mita et al., 2002) allowed us to identify a silkworm homologue of the spodoptericin gene (clone wdS20658), which was added in the alignment (Fig. 2A). A bootstrapped neighbor-joining tree was then generated from the aligned sequences using the same software (Fig. 2B).

When the tree was rooted on the mussel sequence by defining this sequence as an outgroup, the sequences were split into two groups, the scorpion defensins and the other sequences (Fig. 2B). The latter was further split into two subsets, the branching being supported by a high bootstrap value (921 of 1000 replicates). One subset grouped the previously described defensins from neopteran insects (Hemiptera, Coleoptera, Diptera and Hymenoptera) and included the $M$. brassicae defensin. The second subset grouped the cysteine-rich lepidopteran peptides from $S$. frugiperda, $B$. mori and $G$. mellonella including $H$. virescens heliomicin and scorpion toxins (Buthus and Centruroides spp.). Interestingly, spodoptericin and its silkworm homologue appear more related to cobatoxin-like peptides than to gallerimycin peptides, the latter being more related to scorpion cobatoxin than cobatoxin-like peptides themselves. This result was confirmed by the high bootstrap values obtained when only lepidopteran cysteine-rich peptides were analyzed, with heliomicin defined as an outgroup (Fig. 2C).

It thus appears that $S$. frugiperda peptides belong to families of defensin-like molecules that are widespread within the Lepidoptera order. They form a cluster distinct from the previously described insect defensins and are characterized by some degree of similarity, at a sequence level, to short scorpion toxins. As suggested by our tree, $H$. virescens heliomicin is included in this defensin-like group whereas M. brassicae defensin, in spite of five instead of six cysteine residues, is more related to the other neopteran insect defensins. It is noteworthy that two forms of defensins are also present in the diptera Sarcophaga peregrina, with one of the two forms, sapecin-B, also showing structural similarity to potassium-channel-blocking scorpion toxins (Yamada and Natori, 1993). Note that sapecin B groups with the lepidopteran defensin-like peptides in our analysis.

\subsection{Transcription analysis of the S. frugiperda defensin-like genes}

Control experiments showed that all three genes were constitutively expressed in Sf9 cells although at a low level (data not shown). In $S$. frugiperda larvae, in absence of immune challenge, Sf-gallerimycin transcripts are detected during the last larval instar, spodoptericin transcripts during all larval development at low level, and the Sf-cobatoxin gene is essentially transcribed during the two last larval instars (data not shown). The constitutive expression of the Sf-cobatoxin gene is higher than that of the other two genes, particularly in the fat body. In diptera, spontaneous expression of defensin has often been reported (Dimarcq et al., 1994; Lowenberger et al., 1999). As induction of transcription often occurs at the onset of pupal development, it has been suggested that these genes may have a dual role in defense and development (Matsuyama and Natori, 1988).

Since the three $S$. frugiperda genes are putatively antimicrobial genes, we analyzed their response following immune challenge in insects as well as in cells in culture. In insects, transcription was analyzed in two immune tissues of 5 th instar larvae, i.e., hemocytes and fat bodies. Immune challenge consisted in injection of live bacteria or fungi. For bacterial challenge, Gram-negative and Gram-positive bacteria were injected together to ensure activation of one of the two regulation pathways, Toll and Imd, leading to expression of antibacterial genes. The effect of polydnavirus infection was also analyzed since these viruses are immunesuppressive parasitoid wasps symbionts (Webb, 1998). They have been previously shown to inhibit translation, but not transcription, of antibacterial lysozyme genes (Shelby et al., 1998). In all three genes examined, injections of HdIV alone did not induce an increase in transcription (data shown for the spodoptericin, Fig. 3C, panel H, lane V).

After immune challenge, the Sf-gallerimycin gene exhibited a significant up-regulation. Increases in the expression of the two other $S$. frugiperda cysteine-rich genes after a bacterial or fungal challenge are less pronounced, suggesting that they are up-regulated to a lesser extent than the Sf-gallerimycin gene.

\subsubsection{The Sf-gallerimycin gene is significantly up-regulated after bacterial challenge}

In the caterpillar, injection of $E$. coli and B. subtilis induced a strong up-regulation of the Sf-gallerimycin gene $24 \mathrm{~h}$ after immune challenge (Fig. 3A, panels FB and H, lanes B24 and B48). Transcription of this gene occurred essentially in the fat body cells. Conversely, injection of B. bassiana did not significantly increase the level of transcription compared to saline-injected larvae (Fig. 3A, panels FB and H, lanes F24 and F48). A slight increase was observed in the fat body 48 $\mathrm{h}$ post-injection (pi). We thus investigated if this response was related to the dose of conidia injected. We found that, in fat body cells, $24 \mathrm{~h} \mathrm{pi}$, a response was observed for the higher dose only (Fig. 3A, panel FB, lane F $24 \mathrm{e}^{3}$ ) whereas $48 \mathrm{~h}$ pi, 


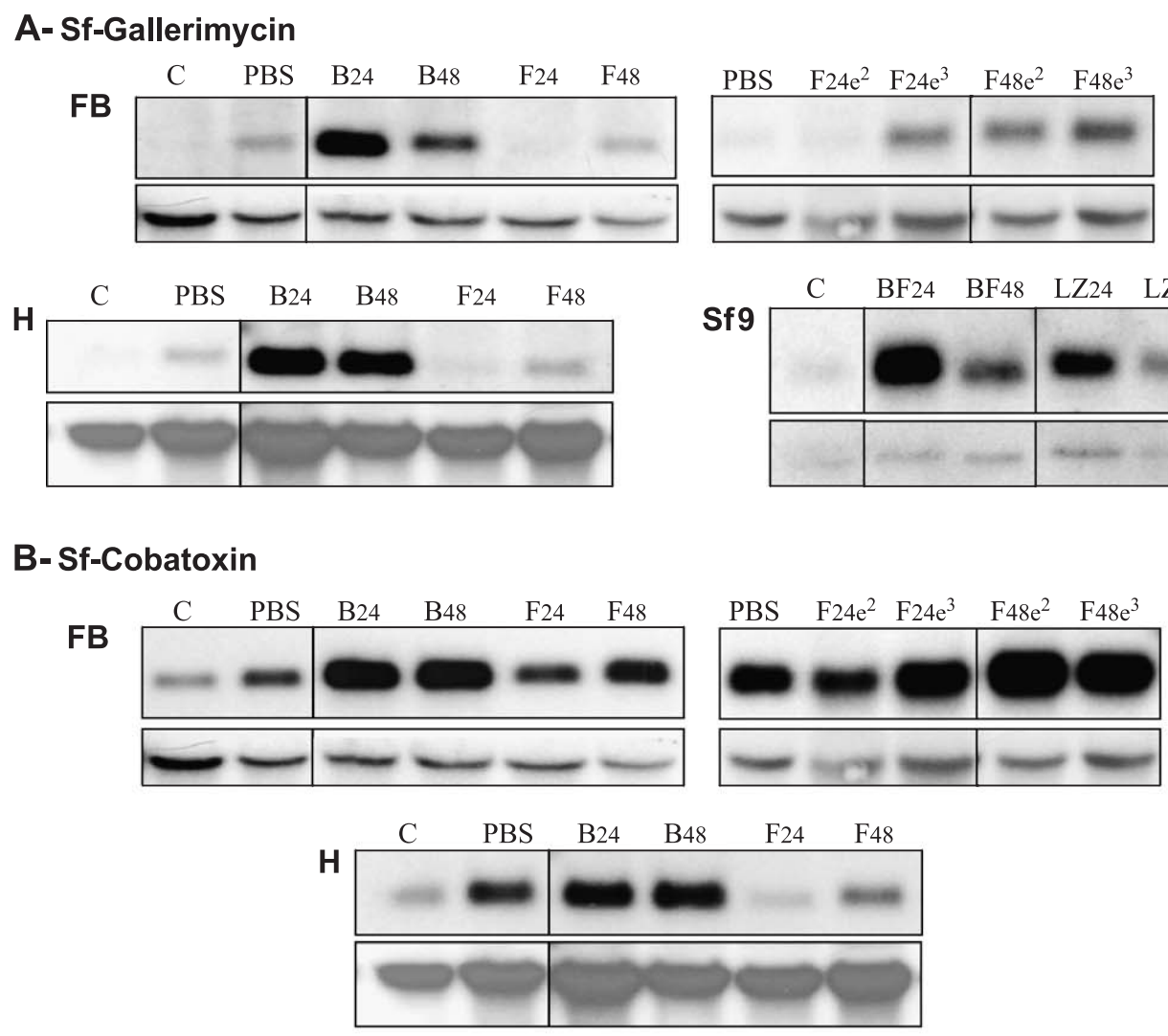

\section{C- Spodoptericin}

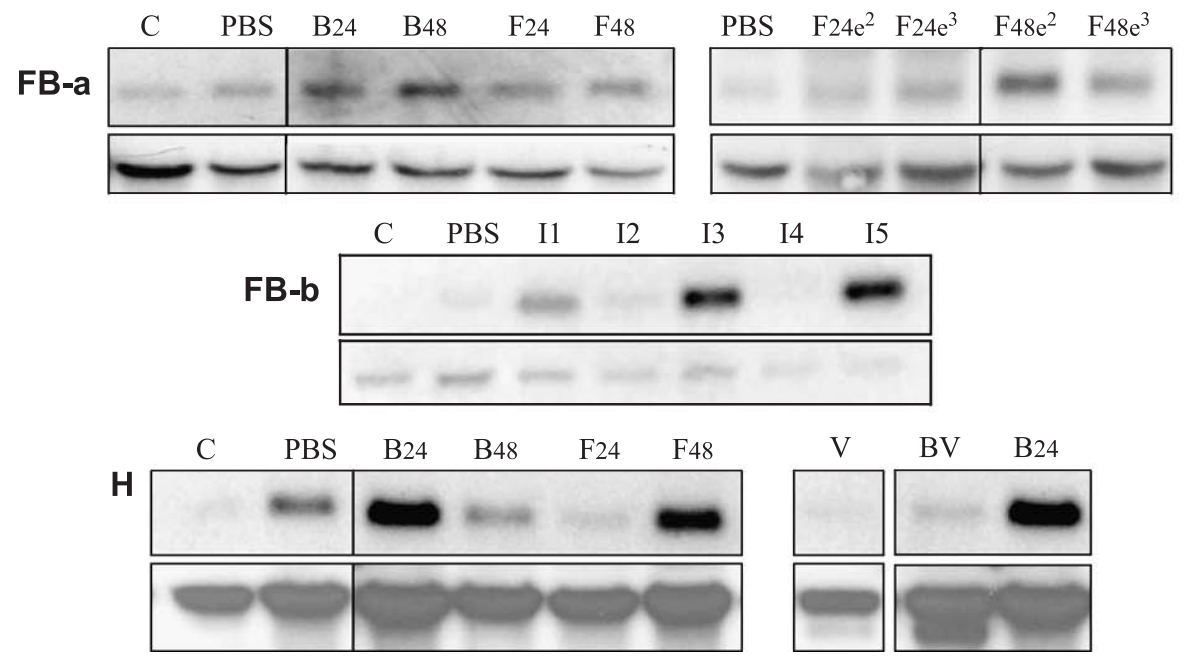

Fig. 3. Effects of immune challenge on transcription of (A) Sf-gallerimycin, (B) Sf-cobatoxin and (C) Spodoptericin. Assays were conducted with Spodoptera frugiperda 5 th instar larvae fat body (panels FB) and hemocytes (panels H). One-day-old larvae were immune-challenged by injection of either a mix of Gram+ and Gram- bacteria (lanes B) or B. bassiana conidia (lanes F). To examine the dose-dependent effects of B. bassiana injection on transcription in the fat body, $8 \times 10^{2}$ (lanes $\mathrm{Fe}^{2}$ ) or $8 \times 10^{3}$ conidia per insect (lanes $\mathrm{Fe}^{3}$ ) were injected. Lanes $\mathrm{C}$ and PBS indicate nonchallenged and PBS-injected controls. All samples were collected and processed 24 (lanes B24 and F24) and 48 (lanes B48 and F48) h postinjection and subsequently hybridized successively with cDNA probes. An actin probe was used to quantify initial amounts of RNA loaded per lane (lower panels). In (A) Sf-gallerimycin, panel Sf9 shows up-regulation of $S f-$ gallerimycin transcripts in Sf9 cells after an immune challenge. Cells were treated either with a suspension of heat-inactivated Gram+, Gram- bacteria and $B$. bassiana conidia (lanes BF) or with a suspension of LPS and zymozan (lanes LZ). Samples were collected 24 and $48 \mathrm{~h}$ after treatment. Lane C indicates the nonchallenged control. In (C) spodoptericin, panel FB-b shows results obtained from individual 5th-instar larvae $48 \mathrm{~h}$ after injection of a mix of bacteria and $B$. bassiana (lanes I1-5). In panel H, results are shown from HdIV-infected larvae without (lane V) or $24 \mathrm{~h}$ after challenge with bacteria (lane BV). 
both doses resulted in higher levels of transcripts (Fig. 3A, panel $\mathrm{FB}$, lanes $\mathrm{F} 48 \mathrm{e}^{2}$ and $\mathrm{F} 48 \mathrm{e}^{3}$ ). It is noteworthy that in natural $B$. bassiana infections, the blastospores and hyphae, and not the conidia, are in direct contact with the hemocoele (Vey and Fargues, 1977). This suggests that the gene upregulation seen here could be related to the presence of the main development stage of $B$. bassiana, which invades the host cavity, e.g., the blastospores.

In immune-challenged Sf9 cells, Sf-gallerimycin transcripts were detected in large amounts $24 \mathrm{~h}$ after presence of a mixture of heat-inactivated bacteria and fungi, or after addition of LPS and zymozan (Fig. 3A, panel Sf9). Induction of antimicrobial peptides in cell lines has previously been reported in insects. For example, upon immune stimulation with heat-killed bacteria, hemocyte-derived mosquito cell lines secrete different classes of immunity factors, including defensins (Fallon and Sun, 2001; Gao et al., 1999).

\subsubsection{The Sf-cobatoxin gene is constitutively expressed and} up-regulated following bacterial challenge in fat body cells

In the S. frugiperda larvae, the Sf-cobatoxin gene is constitutively well expressed, and is up-regulated by saline injection only (Fig. 3B, lane PBS). Note that septic injury by itself is known to induce transcription, albeit at low levels, of antibacterial peptides in Drosophila (Hoffmann and Reichhart, 2002). Following bacterial injection, the Sfcobatoxin gene was up-regulated essentially in the fat body (Fig. 3B, lanes B24 and B48). Hemocyte response to bacterial challenge, if compared to saline injection, was not significantly increased (Fig. 3B, panel H, compare lanes PBS and B24). As for the Sf-gallerimycin gene, injection of B. bassiana induces an increase of Sf-cobatoxin transcription, after $48 \mathrm{~h}$, in the fat body (Fig. 3B, lanes F24 and F48), and response levels appear to be correlated to the dose of injected conidia.

\subsubsection{The spodoptericin gene exhibits low levels of induced transcription after immune challenge}

In the caterpillar fat body, after bacterial challenge, little difference between saline- and germ-injected samples could be seen for spodoptericin transcription (Fig. 3C, panel FB-a, compare lanes PBS and B24, increase of approximately 2fold). These results are consistent with previous reports that defensin genes often exhibit lower levels of infectioninduced transcription compared to other antibacterial peptide genes (Dimarcq et al., 1994). As for the previous two genes, injection of $B$. bassiana induced a slight increase in gene transcription mainly after $48 \mathrm{~h}$. We completed several tests using isolated caterpillars injected with a mix of bacteria and fungi and found a great inter-individual variability in the response (Fig. 3C, panel FB-b). This could explain a lower overall response when pooled larvae are analyzed.

In hemocytes, injection of bacteria led to an increase in the number of spodoptericin transcripts, although this increase is not clearly significant and may be related to a larger amount of loaded RNA (Fig. 3C, compare lanes PBS and B24, spodoptericin and actin probes). Conversely to the other two genes, we found a strong hybridization signal in the hemocytes $48 \mathrm{~h}$ after injection of fungi (Fig. 3C, panel $\mathrm{H}$, lane F48). Also in contrast to the other two genes (data not shown), HdIV infection of the hemocytes appeared to inhibit full inducibility of the spodoptericin gene (Fig. 3C, panel $\mathrm{H}$, compare lanes BV and B24). Thus, our data suggest that, in addition to their known effects on cellular immune response (Webb, 1998), polydnaviruses may also affect, to some extent, host gene transcription.

To conclude on the transcription of the three $S$. frugiperda defensin-like genes, the Sf-gallerimycin and Sf-cobatoxin are up-regulated, to variable degrees, mainly in the fat body cells, and essentially in response to a bacterial infection. The spodoptericin gene also undergoes up-regulation although it is not as prominent. In vitro, the induction of the spodoptericin and the Sf-cobatoxin genes was not detected (data not shown) but we found induction of the Sf-gallerimycin gene. This observation suggests that the necessary circulating receptors or pathogen recognition proteins known to be required for induction of antibacterial peptide genes (Choe et al., 2002; Michel et al., 2001) are present in vitro and that Sf9 cells contain sufficient amounts of specific necessary transcription factors. Indeed, differential regulation of Rel proteins, for example, can lead to preferential expression of specific target genes (Han and Ip, 1999).

\subsection{Structure of the S. frugiperda genes encoding defensin- like peptides}

To date, genomic data on defensin genes are still scarce and most of our knowledge pertains to dipteran genes. As opposed to genomes of flies and mosquitoes, arthropod genomes are still poorly characterized. Furthermore, most known peptides in arthropods have been identified from immune-challenged hemolymph by mass spectrometry so that no information on nucleic acid sequences is available. This led us to further characterize the $S$. frugiperda defensin-like genes since it should provide valuable information on this gene family.

\subsubsection{Gene copy number in the S. frugiperda genome}

Our hybridization results strongly suggest that the spodoptericin and Sf-gallerimycin genes are encoded by a single copy sequence (data not shown). Southern blot analysis of PstI digested $S$. frugiperda DNA resulted in unique hybridization bands of $\sim 10$ and $\sim 8 \mathrm{kbp}$ for the spodoptericin and Sf-gallerimycin, respectively. This result was confirmed by probing our $10 \times$ coverage BAC library with the spodoptericin coding sequence, resulting in 11 positive spots. Similar experiment on half of the BAC library resulted in seven positive spots for the Sf-gallerimycin probe. A single gene copy is also present in the Drosophila (Dimarcq et al., 1994) and the Anopheles gambiae (Eggleston et al., 2000) genomes, whereas Aedes aegypti displays two copies of this gene 
(Lowenberger et al., 1999). Because of unexplained additional hybridization bands, no conclusions could be drawn for the Sf-cobatoxin gene.

\subsubsection{Splicing}

The Drosophila defensin gene displays no introns (Dimarcq et al., 1994) whereas one short intron is present in A. gambiae (Eggleston et al., 2000) and A. aegypti (Cho et al., 1997) genes (Fig. 4, upper diagram). In S. frugiperda, alignment of cDNA and PCR-amplified genomic sequences showed that the spodoptericin, Sf-gallerimycin and Sf-cobatoxin genes all contain one intron (597, 465 and $822 \mathrm{nt}$ long, respectively) located within the ORF (GenBank \# AY128091, AY236867 andAY236868), 8, 18 and 19 aa before the cysteine motif (Fig. 4, upper diagram). Note that $A$. gambiae and $A$. aegypti introns are located 21 aa upstream the cysteine motif. The positions within the coding sequence and length of introns are thus quite variable among the three $S$. frugiperda genes and differ from the mosquitoes defensin genes. Interestingly, in the Sf-cobatoxin gene, the 3'sequence shared by the SF2701 and SF6099 clones starts with the beginning of the second exon. Since the SF6099 sequence was amplified by PCR, it could be hypothesized that the RNA corresponding to SF2701 results from alternative splicing, the corresponding first exon being upstream from the SF6099 sequence. However, attempts to amplify a genomic sequence by PCR using a forward primer in the SF2701 sequence (46 nt upstream the SF2701 putative ATG codon) failed. Thus, because two Sf-cobatoxin cDNAs exist and share the second exon sequence, only one RNA is detected in Northern blot, and hybridization with genomic DNA leads to inconclusive results, the genomic structure of Sf-cobatoxin and the hypothesis of an alternative splicing will be further investigated in the near future.

\subsection{3. cDNA upstream region analysis}

Partial promoter sequences were analyzed for the wellinduced Sf-gallerimycin gene (GenBank \# AY236867) and the poorly induced spodoptericin gene (GenBank \# AY128091). In the Sf-gallerimycin sequence, a putative TATA box is located $26 \mathrm{nt}$ upstream from the start of the cDNA sequence. Binding sequences for transcription factors known to be involved in the immune response were identified. A clear consensus NF- $\kappa$ B binding site was found 250 bp upstream from the cDNA first nucleotide (nt) (Fig. 4A). Several GATA-binding factor 1 (GATA-1) motifs were also identified, as well as two binding sites for interleukin-6 response element binding protein (IL-6 RE-BP) (Fig. 4A). For the spodoptericin gene, the only found TATA box sequence was located in the Sf9 cDNA 5'UTR. In contrast to the Sf-gallerimycin gene, no clear consensus binding site was found for NF- $\kappa$ B transcription factor (Fig. 4B). A few low conserved GATA-1 and two dorsal putative binding sites were found approximately $500 \mathrm{bp}$ upstream from the cDNA start (Fig. 4B). Interestingly, the upstream region contains a 39-nt imperfectly repeated sequence, which includes a 10-nt conserved motif (Fig. 4B). TFSEARCH analysis of the complete gene, including introns, did not reveal other relevant transcription factor binding sites that could suggest how this gene is regulated. All three genes as well as the Drosophila and Anopheles defensin genes present several sites of factors possibly involved in development [for example, octamer-binding factor 1 (Oct-1), Brn-2, c-Myb, Deformed (Dfd), CdxA], but we are, at this point, unable to clearly interpret these data.

Thus, the two $S$. frugiperda defensin-like genes differ by their promoter sequences as well as by their response to immune challenge. Because of the presence of known cis regulatory elements, Sf-gallerimycin transcription is likely to be regulated by the classical pathways, Toll and/or Imd, known to control Rel/NF-kB-like mediated immune antimicrobial response in insects. On the other hand, the absence of such consensus binding sites in the 700-nt upstream sequence of spodoptericin might indicate that the regulation of the transcription of this gene, which occurs at low level, depends on these pathways, and in this case, binding sites are more distant from the coding sequence, or depends on other pathways and/or other unknown transcription factors. Indeed, a number of unresolved questions in insect innate immunity remain and the possible existence of a third pathway, distinct from the known Imd and Toll pathways, has been proposed even in the well-known Drosophila immunity model (Hoffmann and Reichhart, 2002).

\subsection{Conclusion}

1. To our knowledge, this work is the first report on genomic and transcriptional characteristics of lepidopteran cysteine-rich defensin-like genes in fat bodies and hemocytes.

2. Three defensin-like $S$. frugiperda genes are described. Spodoptericin is a novel putative anionic peptide that exhibits low levels of induced transcription after immune challenge. In contrast, Sf-gallerimycin is a putative cationic peptide that is up-regulated in both immune tissues and $\mathrm{Sf} 9$ cells following immune challenge. Sf-cobatoxin is a putative cationic peptide, which exhibits high levels of constitutive transcription and is up-regulated in immune tissues following immune challenge.

3. These defensin-like genes are widespread in lepidopterans, with homologues found in at least two other species. They differ from previously described neoptera insect defensins and are characterized by their similarity with short scorpion toxins. This suggests the existence of two types of defensins in lepidoptera-as previously described in S. peregrina - one type similar to neoptera insect defensin (such as the M. brassicae defensin), the other more similar to short scorpion toxins.

4. On the basis of information provided by the partial promoter sequence analyses, the Sf-gallerimycin transcription could be regulated by the classical pathways known to control Rel/NF-kB-like mediated immune 


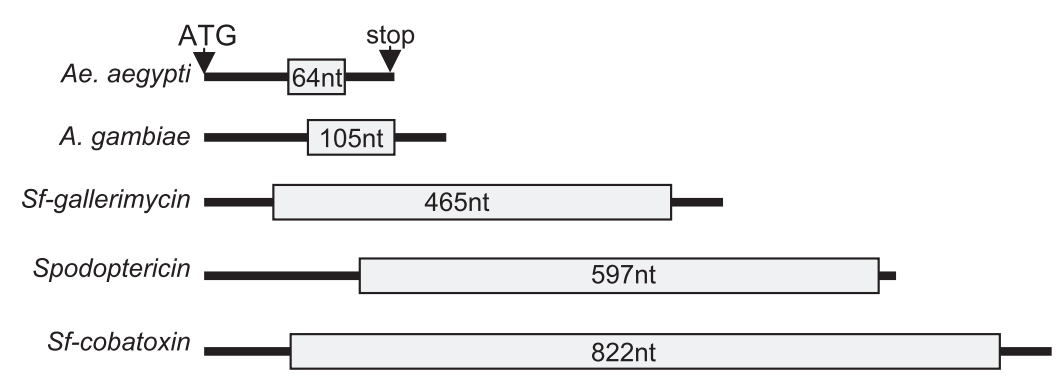

A. Upstream sequence of the Sf-gallerimycin gene

ccaatcccactcataaaaacacatatcagtatatccttaatacactgtaatcctaatttacttagataag

aattacatactgtatattgaataattataaatgcaaaattaacaggaaatgaatgacaaacattgtaaa 144

ctgaatatttacctctaacccacagataacaagaacatacaatgataactgttcacaataccaacaaacat 216

caccctgtttaagattcatacaaacaaggataatcaactttaatctcgttctgatatagtggtaaattga 288

atgacgaatacggaatgtatcacttgcaattctatgccagaacagggactcagggtattccctccacataca 360

$\mathrm{NF}-\mathrm{B}(+)$

atattaatgtggtagcgtatagtgcgaagcgactgattgcacgggactgtttctgcggtatccatttccgtc 432

tgaacacgactgtaaatacgttacgagaatagtttagcagcaatgtatgtttaagtgaaatgtatttagatt 504 IL6-RE

tggtcgtgttgtttatcgatctggtggtatagtttgggtgaagttatacgtgggtctgagttagtataaata 576

[Sf9 cDNA

aggtggagctggaaagtccgcacacattgaaaggtttcagtc ATG AAG GCT TGC GTG GTT CTC 639

$\begin{array}{lllllllll}M & \mathrm{~K} & \mathrm{~A} & \mathrm{C} & \mathrm{V} & \mathrm{V} & \mathrm{L} & 7\end{array}$

B. Upstream sequence of the Spodoptericin gene:

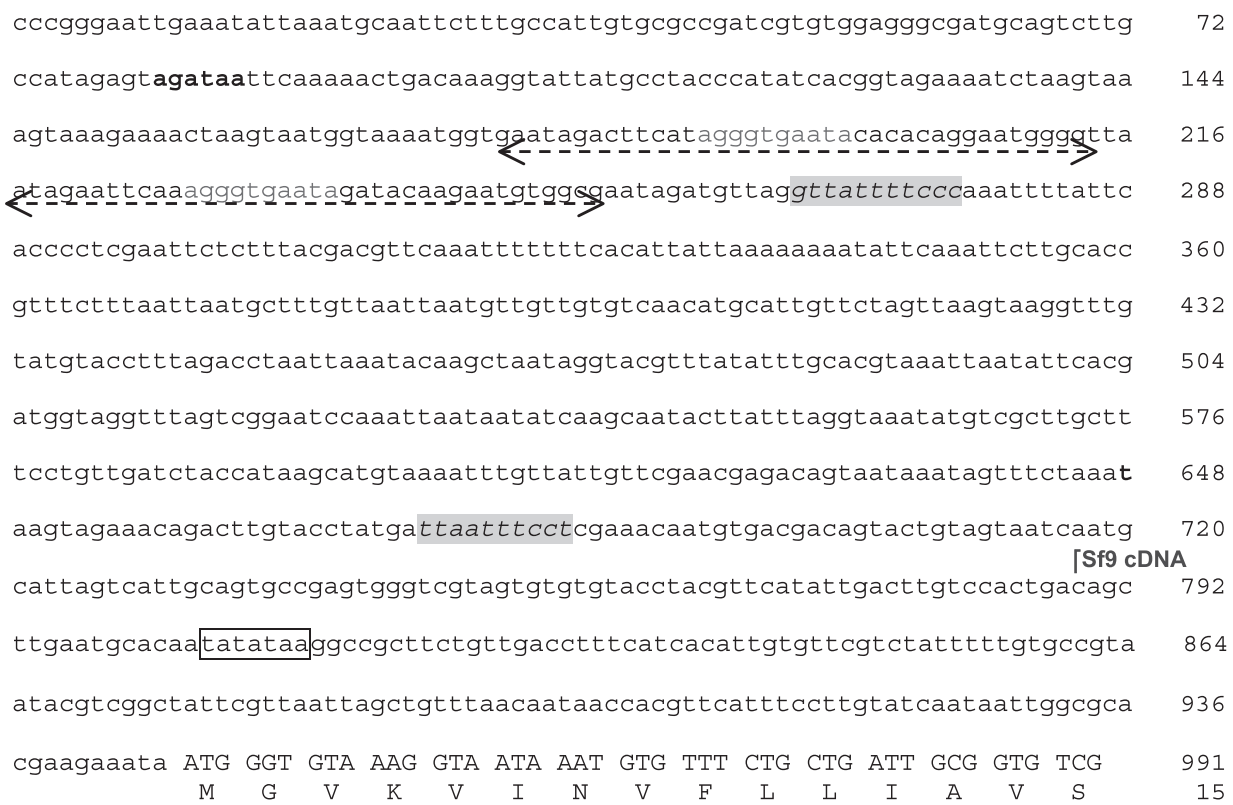

Fig. 4. Genomic sequence analysis. The upper diagram shows intron position and length within the three $S$. frugiperda coding sequences compared to $A$. aegypti and A. gambiae defensin genes. (A) Sf-gallerimycin upstream sequence (AY236867). (B) Spodoptericin upstream sequence (AY128091). Start of cDNA sequences is indicated above sequence. Putative TATA boxes are boxed. NF- $\mathrm{B}$ consensus binding sites are highlighted; italics indicate a weak consensus. Predicted GATA-1 binding sites are in bold characters, and predicted IL6-RE are underlined. In the spodoptericin gene, horizontal arrows limit the two tandem repeats, the conserved $10 \mathrm{nt}$ are in grey.

antimicrobial response in insects whereas regulation of the spodoptericin gene may rely on other unknown transcription factors.
5. Although the assessment of the antimicrobial activity of the three S. frugiperda peptides will require their purification and assays of inhibition of bacteria and 
fungi growth, transcriptional analyses suggest that these genes do encode for antimicrobial peptides.

\section{Acknowledgements}

The authors are very grateful to Charles Hetru, Matt Turnbull and Hélène Volkoff for their comments on the manuscript. We thank Bertrand Limier for providing the $S$. frugiperda larvae, and Simone Berger for her technical assistance.

\section{References}

Cho, W., Fu, T., Chiou, J., Chen, C., 1997. Molecular characterization of a defensin gene from the mosquito Aedes aegypti. Insect Biochemistry and Molecular Biology 27, 351-358.

Choe, K., Werner, T., Stöven, S., Hultmark, D., Anderson, K.V., 2002. Requirement for a peptidoglycan recognition protein (PGRP) in relish activation and antibacterial immune response in Drosophila. Science 296, 359-362.

Cociancich, S., Ghazi, A., Hetru, C., Hoffmann, J.A., Letellier, L., 1993. Insect defensin, an inducible antibacterial peptide, forms voltage-dependent channels in Micrococcus luteus. Journal of Biological Chemistry $26,19239-19245$.

Dimarcq, J.L., Hoffmann, D., Meister, M., Bulet, P., Lanot, R., Reichhart, J.M., Hoffmann, J.A., 1994. Characterization and transcriptional profiles of a Drosophila gene encoding an insect defensin. European Journal of Biochemistry 221, 201-209.

Dimarcq, J.-L., Bulet, P., Hetru, C., Hoffmann, J., 1998. Cysteine-rich antimicrobial peptides in Invertebrates. Biopolymers (Peptide Science) 47, 465-477.

Eggleston, P., Lu, W., Zhao, Y., 2000. Genomic organization and immune regulation of the defensin gene from the mosquito, Anopheles gambiae. Insect Molecular Biology 9, 481-490.

Fallon, A.M., Sun, D., 2001. Exploration of mosquito immunity using cells in culture. Insect Biochemistry and Molecular Biology 31, 263-278.

Gao, Y., Hernandez, V.P., Fallon, A.M., 1999. Immunity proteins from mosquito cell lines include three defensin A isoforms from Aedes aegypti and a defensin D from Aedes albopictus. Insect Molecular Biology $8,311-318$

Han, Z.S., Ip, Y.T., 1999. Interaction and specificity of Rel-related proteins in regulating Drosophila immunity gene expression. The Journal of Biological Chemistry 274, 21355-21361.

Hoffmann, J.A., Reichhart, J.M., 2002. Drosophila innate immunity: an evolutionary perspective. Nature Immunology 3, 121-126.

Hoffmann, J.A., Kafatos, F.C., Janeway Jr., C.A., Ezekowitz, R.A.B., 1999. Phylogenetic perspectives in innate immunity. Science 284, 1313-1318.

Khush, R.S., Leulier, F., Lemaitre, B., 2001. Drosophila immunity: two paths to NF-KB. Trends in Immmunology 22, 260-264.

Lamberty, M., Ades, S., Uttenweiler-Joseph, S., Brookhart, G., Bushey, D., Hoffmann, J.A., Bulet, P., 1999. Isolation from the lepidopteran Heliothis virescens of a novel insect defensin with potent antifungal activity. The Journal of Biological Chemistry 274, 9320-9326.
Landais, I., Ogliastro, M., Mita, K., Nohata, J., Lopez-Ferber, M., DuonorCérutti, M., Shimada, T., Fournier, P., Devauchelle, G., 2003. Annotation pattern of ESTs from Spodoptera frugiperda Sf9 cells and analysis of the ribosomal protein genes reveal insect-specific features and unexpectedly low codon usage bias. Bioinformatics (in press).

Lemaitre, B., Reichhart, J.M., Hoffmann, J.A., 1997. Drosophila host defense: differential induction of antimicrobial peptide genes after infection by various classes of microorganisms. Proceedings of the National Academy of Sciences of the United States of America 94, 14614-14619.

Lowenberger, C.A., Smartt, C.T., Bulet, P., Ferdig, M.T., Severson, D.W., Hoffmann, J.A., Christensen, B.M., 1999. Insect immunity: molecular cloning, expression, and characterization of cDNAs and genomic DNA encoding three isoforms of insect defensin in Aedes aegypti. Insect Molecular Biology 8, 107-118.

Matsuyama, K., Natori, S., 1988. Molecular cloning of cDNA for Sapecin and unique expression of the sapecin gene during the development of Sarcophaga peregrina. The Journal of Biological Chemistry 263, 17117-17121.

Meister, M., Braun, A., Kappler, C., Reichhart, J.M., Hoffmann, J.A., 1994. Insect immunity. A transgenic analysis in Drosophila defines several functional domains in the diptericin promoter. EMBO Journal 13, $5958-5966$.

Michel, T., Reichhart, J.M., Hoffmann, J.A., Royet, J., 2001. Drosophila Toll is activated by Gram-positive bacteria through a circulating peptidoglycan recognition protein. Nature 414, 756-759.

Mita, K., Morimyo, M., Okano, K., Koike, Y., Nohata, J., Suzuki, M.G., Shimada, T., 2002. Construction of an EST database for Bombyx mori and its applications. Current Science 83, 426-431.

Ponnuvel, K.M., Yamakawa, M., 2002. Immune responses against bacterial infection in Bombyx mori and regulation of host gene expression. Current Science 83, 447-454.

Seitz, V., Clermont, A., Wedde, M., Hummel, M., Vilcinskas, A., Schlatterer, K., Podsiadlowski, L., 2003. Identification of immunorelevant genes from greater wax moth (Galleria mellonella) by a substractive hybridization approach. Developmental and Comparative Immunology 27, 207-215.

Shelby, K.S., Cui, L., Webb, B.A., 1998. Polydnavirus-mediated inhibition of lysozyme gene expression and the antibacterial response. Insect Molecular Biology 7, 265-272.

Vey, A., Fargues, J., 1977. Histological and ultrastructural studies of Beauveria bassiana infection in Leptinotarsa decemlineata larvae during ecdysis. Journal of Invertebrate Pathology 30, 207-215.

Volkoff, A.-N., Cerutti, P., Rocher, J., Ohresser, M.C., Devauchelle, G., Duonor-Cerutti, M., 1999. Related RNAs in lepidopteran cells after in vitro infection with Hyposoter didymator virus define a new polydnavirus gene family. Virology 263, 349-363.

Volkoff, A.-N., Béliveau, C., Rocher, J., Levasseur, A., Duonor-Cérutti, M., Cusson, M., Webb, B.A., 2002. Evidence for a conserved polydnavirus gene family: ichnovirus homologs of the CsIV repeat element genes. Virology 300, 316-331.

Webb, B.A., 1998. Polydnavirus biology, genome structure, and evolution. In: Miller, L.K., Balls, L.A. (Eds.), The Insect Viruses. Plenum, New York, pp. 105-139.

Yamada, K., Natori, S., 1993. Purification, sequence and antibacterial activity of two novel sapecin homologues from Sarcophaga embryonic cells: similarity of sapecin B to charybdotoxin. Biochemical Journal 291, 275-279. 